\title{
LARGE SUPER-FAST ROTATOR HUNTING USING THE INTERMEDIATE PALOMAR TRANSIENT FACTORY
}

\author{
Chan-Kao Chang ${ }^{1}$, Hing-Wen Lin ${ }^{1}$, Wing-Huen IP ${ }^{1,2}$, Thomas A. Prince ${ }^{3}$, Shrinivas R. Kulkarni ${ }^{3}$, David Levitan ${ }^{3}$, \\ RuSS LAHER $^{4}$, AND JASON Surace ${ }^{4}$ \\ ${ }^{1}$ Institute of Astronomy, National Central University, Jhongli, Taiwan; rex @astro.ncu.edu.tw \\ ${ }^{2}$ Space Science Institute, Macau University of Science and Technology, Macau \\ ${ }^{3}$ Division of Physics, Mathematics and Astronomy, California Institute of Technology, Pasadena, CA 91125, USA \\ ${ }^{4}$ Spitzer Science Center, California Institute of Technology, M/S 314-6, Pasadena, CA 91125, USA \\ Received 2016 June 26; revised 2016 August 17; accepted 2016 August 25; published 2016 December 2
}

\begin{abstract}
In order to look for large super-fast rotators, in late 2014 and early 2015, five dedicated surveys covering $\sim 188 \mathrm{deg}^{2}$ in the ecliptic plane have been carried out in the $R$-band, with $\sim 10$ minute cadence using the intermediate Palomar Transient Factory. Among 1029 reliable rotation periods obtained from the surveys, we discovered 1 new large super-fast rotator, (40511) 1999 RE88, and 18 other candidates. (40511) 1999 RE88 is an S-type inner main-belt asteroid with a diameter of $D=1.9 \pm 0.3 \mathrm{~km}$, a rotation period of $P=1.96 \pm 0.01 \mathrm{hr}$, and a light curve amplitude of $\Delta m \sim 1.0 \mathrm{mag}$. To maintain such fast rotation, an internal cohesive strength of $\sim 780 \mathrm{~Pa}$ is required. Combining all known large super-fast rotators, their cohesive strengths all fall in the range of 100-1000 Pa of lunar regolith. However, the number of large super-fast rotators seems to be far less than the whole asteroid population. This might indicate a peculiar asteroid group for them. Although the detection efficiency for a long rotation period is greatly reduced due to our two-day observation time span, the spin-rate distributions of this work show consistent results with Chang et al. (2015), after considering the possible observational bias in our surveys. It shows a number decrease with an increase of spin rate for asteroids with a diameter of $3 \leqslant D \leqslant 15 \mathrm{~km}$, and a number drop at a spin rate of $f=5 \mathrm{rev} \mathrm{day}^{-1}$ for asteroids with $D \leqslant 3 \mathrm{~km}$.
\end{abstract}

Key words: minor planets, asteroids: general - surveys

Supporting material: extended figures, machine-readable tables

\section{INTRODUCTION}

It was once a formidable task to collect a large number of asteroid rotation periods, but it is becoming easier. With advances in observational technology, several data sets containing hundreds to thousands of asteroid rotation periods have been acquired through large sky surveys (Masiero et al. 2009; Polishook \& Brosch 2009; Dermawan et al. 2011; Polishook et al. 2012; Chang et al. 2014a, 2015). Moreover, numerous asteroid rotation periods, obtained from various time-series-archived data products (see an example of Waszczak et al. 2015), and single target observations from the Asteroid Light Curve Database (LCDB; Warner et al. 2009), ${ }^{5}$ also provide major contributions to this field. Therefore, a more comprehensive understanding of asteroid rotations has emerged and possible applications could be conducted as well (see, e.g., Chang et al. 2016).

While the spin-rate distributions obtained from large skycoverage surveys and archived data show a number decrease with spin rate at frequencies $f>5 \mathrm{rev} \mathrm{day}^{-1}$ (Masiero et al. 2009; Chang et al. 2015; Waszczak et al. 2015), a flat distribution was indicated by the single target observations (Pravec et al. 2008, update 2014-04-20). However, the tendency of number decrease still remains in the asteroid spin-rate distributions from the large sky surveys after taking into account possible observational bias (Masiero et al. 2009; Chang et al. 2015). In addition, the spin-rate distribution of asteroids with diameters of $D \leqslant 3 \mathrm{~km}$ seems to have a number

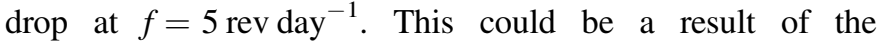
Yarkovsky-O'Keefe-Radzievskii-Paddack effect (YORP; Rubincam 2000), which works relatively fast on small asteroids

\footnotetext{
5 http://www.minorplanet.info/lightcurvedatabase.html
}

and pushes more of them over the spin barrier to break up (Chang et al. 2015).

The "spin barrier" at $2.2 \mathrm{hr}$ (Harris 1996; Pravec et al. 2002) is persistently seen in these data sets. Furthermore, with sufficiently large samples, the C-type asteroids were for the first time found to show a rotation-period limit that was longer than that of the S-type asteroids (Chang et al. 2015; Waszczak et al. 2015). This is in accordance with the general picture for rubble-pile asteroids that: (a) they cannot rotate exceedingly fast; and (b) those with lower bulk density should have a longer rotation-period limit $(P \sim 3.3 \sqrt{(1+\Delta m) / \rho}$; Harris 1996). However, the presence of four large (i.e., a few hundred meters) super-fast rotators (hereafter, SFRs), 2001 OE84 (Pravec et al. 2002), 2005 UW163 (Chang et al. 2014b), 1950 DA(Rozitis et al. 2014), and 2000 GD65 (Polishook et al. 2016), suggest that internal cohesion might be required to keep them from breaking apart (Holsapple 2007; Sánchez \& Scheeres 2012). Compared to the majority of large asteroids with known rotation periods, the number of detected large SFRs seems to be very small. Therefore, a comprehensive census of the population of large SFRs should provide key information regarding asteroid interior structure.

Five asteroid rotation-period surveys were carried out to look for large SFRs. We obtained 7984 asteroid light curves with detections $\geqslant 10$, from which 1029 reliable rotation periods were derived. Among them, we discovered one new large SRF, (40511) 1999 RE88, and 18 other candidates. The observation information and method of light curve extraction are given in Section 2. The rotation-period analysis is described in Section 3. The results and discussion are provided in Section 4. A summary and our conclusions are presented in Section 5. 


\section{OBSERVATIONS AND DATA REDUCTION}

To explore the the transient and variable sky synoptically, the PTF/iPTF employs the Palomar 48-inch Oschin Schmidt Telescope, which is equipped with an 11-chip mosaic CCD camera (note that the 11th chip went out of service in early 2015 , so there were therefore 10 available chips at that time) to create a field of view of $\sim 7.26 \mathrm{deg}^{2}$ and a pixel scale of 1 "'01 (Law et al. 2009; Rau et al. 2009). The available filters include the Mould $R$ band, with which most exposures were taken, Gunn- $g^{\prime}$, and two different $H_{\alpha}$-bands. The exposure time is fixed at 60 seconds, which can reach a median limiting magnitude of $R \sim 21 \mathrm{mag}$ at the $5 \sigma$ level (Law et al. 2010).

All PTF/iPTF exposures are processed by the IPAC-PTF photometric pipeline (Grillmair et al. 2010; Laher et al. 2014), and the absolute magnitude, calibrated against Sloan Digital Sky Survey fields (hereafter, SDSS; York et al. 2000), can routinely reach a precision of $\sim 0.02$ mag on photometric nights (Ofek et al. 2012a, 2012b). Since the magnitude calibration is based on a per-night, per-filter, per-chip basis, small photometric zero-point variations are present in catalogs for different nights, fields, filters, and chips.

In order to look for large SFRs, we conducted five asteroid rotation-period surveys during 2014 October 29-31 and November 10-13, and 2015 January 18-19, February 20-21 and 25-26. Each survey continuously scanned six consecutive PTF fields over the ecliptic plane in the $R$-band, with a cadence of 10 minutes. While the first two surveys in late 2014 were observed over the course of three consecutive nights, the last three were observed for only two consecutive nights. However, there were only a few exposures in the first and last nights for the 2014 November observations, due to bad weather conditions. We ended up with a total sky coverage of $\sim 188 \mathrm{deg}^{2}$. The observational metadata are listed in Table 1 .

To extract the light curves of known asteroids, we removed the stationary sources from the catalogs and then matched the detections against the ephemerides obtained from the $J P L$ HORIZONS system with a search radius of $2^{\prime \prime}$. We also excluded the detections flagged as a defect by the IPAC-PTF photometric pipeline from the light curves. Finally, there were 7914 asteroid light curves with a number of detections $\geqslant 10$ (hereafter, PTF-detected asteroids) for the rotation-period analysis described in the next section.

\section{ROTATION-PERIOD ANALYSIS}

All the measurements in the light curves were corrected for light-travel time and reduced to both heliocentric, $r$, and geocentric, $\Delta$, distances at $1 \mathrm{au}$. Since the changes of the phase angles are small for our observational time span, we simply estimated the absolute magnitude by applying a fixed $G_{R}$ slope of 0.15 in the $H-G$ system (Bowell et al. 1989). Then, we followed the traditional second-order Fourier series method to derive the rotation period (Harris et al. 1989):

$$
\begin{aligned}
M_{i, j}= & \sum_{k=1,2}^{N_{k}} B_{k} \sin \left[\frac{2 \pi k}{P}\left(t_{j}-t_{0}\right)\right] \\
& +C_{k} \cos \left[\frac{2 \pi k}{P}\left(t_{j}-t_{0}\right)\right]+Z_{i},
\end{aligned}
$$

where $M_{i, j}$ are the $R$-band reduced magnitudes measured at the light-travel time corrected epoch, $t_{j} ; B_{k}$ and $C_{k}$ are the Fourier coefficients; $P$ is the rotation period; and $t_{0}$ is an arbitrary
Table 1

\begin{tabular}{|c|c|c|c|c|c|}
\hline Field ID & $\begin{array}{l}\text { R.A. } \\
\left({ }^{\circ}\right)\end{array}$ & $\begin{array}{c}\text { Decl. } \\
\left({ }^{\circ}\right)\end{array}$ & $\begin{array}{c}2014 \text { Oct } 29 \\
\Delta t, N_{\exp }\end{array}$ & $\begin{array}{c}2014 \text { Oct } 30 \\
\Delta t, N_{\exp }\end{array}$ & $\begin{array}{c}2014 \text { Oct } 31 \\
\Delta t, N_{\exp }\end{array}$ \\
\hline 3019 & 25.71 & 7.88 & $4.1,16$ & $4.8,28$ & $3.2,20$ \\
\hline 3124 & 25.96 & 10.12 & $4.1,17$ & $4.8,28$ & $3.0,19$ \\
\hline 3125 & 29.42 & 10.12 & $2.7,11$ & $4.8,28$ & $3.2,20$ \\
\hline 3228 & 26.21 & 12.38 & $4.1,12$ & $4.8,28$ & $3.0,19$ \\
\hline 3229 & 29.71 & 12.38 & $4.0,15$ & $4.8,28$ & $3.2,20$ \\
\hline 3332 & 30.00 & 14.62 & $3.8,13$ & $4.8,28$ & $3.2,20$ \\
\hline Field ID & $\begin{array}{l}\text { R.A. } \\
\left({ }^{\circ}\right)\end{array}$ & $\begin{array}{l}\text { Decl. } \\
\left({ }^{\circ}\right)\end{array}$ & $\begin{array}{c}2014 \text { Nov } 10 \\
\Delta t, N_{\exp }\end{array}$ & $\begin{array}{l}2014 \text { Nov } 11 \\
\Delta t, N_{\exp }\end{array}$ & $\begin{array}{c}2014 \text { Nov } 13 \\
\Delta t, N_{\exp }\end{array}$ \\
\hline 3125 & 29.42 & 10.12 & $1.6,7$ & $5.0,30$ & $1.0,6$ \\
\hline 3229 & 29.71 & 12.38 & $1.7,9$ & $5.0,30$ & $2.1,6$ \\
\hline 3230 & 33.20 & 12.38 & $1.8,9$ & $5.0,30$ & $0.3,3$ \\
\hline 3332 & 30.00 & 14.62 & $1.7,8$ & $5.0,30$ & $1.5,6$ \\
\hline 3333 & 33.53 & 14.62 & $1.7,8$ & $5.0,30$ & $1.9,6$ \\
\hline 3435 & 33.86 & 16.88 & $1.7,8$ & $5.0,30$ & $0.3,3$ \\
\hline Field ID & \multicolumn{2}{|c|}{$\begin{array}{l}\text { R.A. } \\
\left({ }^{\circ}\right)\end{array}$} & $\begin{array}{l}\text { Decl. } \\
\left({ }^{\circ}\right)\end{array}$ & $\begin{array}{c}2015 \text { Jan } 18 \\
\Delta t, N_{\exp }\end{array}$ & $\begin{array}{c}2015 \text { Jan } 19 \\
\Delta t, N_{\exp }\end{array}$ \\
\hline 3559 & \multicolumn{2}{|c|}{117.00} & 19.12 & $5.7,34$ & $5.9,36$ \\
\hline 3560 & \multicolumn{2}{|c|}{120.60} & 19.12 & $5.7,34$ & $5.9,36$ \\
\hline 3561 & \multicolumn{2}{|c|}{124.20} & 19.12 & $5.7,34$ & $5.9,36$ \\
\hline 3562 & \multicolumn{2}{|c|}{127.80} & 19.12 & $5.7,33$ & $5.9,36$ \\
\hline 3563 & \multicolumn{2}{|c|}{131.40} & 19.12 & $5.7,34$ & $5.9,36$ \\
\hline 3564 & \multicolumn{2}{|c|}{135.00} & 19.12 & $5.7,35$ & $5.9,36$ \\
\hline Field ID & \multicolumn{2}{|c|}{$\begin{array}{l}\text { R.A. } \\
\left({ }^{\circ}\right)\end{array}$} & $\begin{array}{l}\text { Decl. } \\
\left({ }^{\circ}\right)\end{array}$ & $\begin{array}{c}2015 \text { Jan } 20 \\
\Delta t, N_{\exp }\end{array}$ & $\begin{array}{c}2015 \operatorname{Jan} 21 \\
\Delta t, N_{\exp }\end{array}$ \\
\hline 3461 & \multicolumn{2}{|c|}{126.53} & 16.88 & $6.1,35$ & $5.5,34$ \\
\hline 3462 & \multicolumn{2}{|c|}{130.10} & 16.88 & $6.2,35$ & $5.5,34$ \\
\hline 3463 & \multicolumn{2}{|c|}{133.66} & 16.88 & $6.2,35$ & $5.5,34$ \\
\hline 3464 & \multicolumn{2}{|c|}{137.23} & 16.88 & $6.2,36$ & $5.5,34$ \\
\hline 3465 & \multicolumn{2}{|c|}{140.79} & 16.88 & $6.2,36$ & $5.5,34$ \\
\hline 3466 & \multicolumn{2}{|c|}{144.36} & 16.88 & $6.2,36$ & $5.5,34$ \\
\hline Field ID & \multicolumn{2}{|c|}{$\begin{array}{l}\text { R.A. } \\
\left({ }^{\circ}\right)\end{array}$} & $\begin{array}{c}\text { Decl. } \\
\left({ }^{\circ}\right)\end{array}$ & $\begin{array}{c}2015 \text { Feb } 25 \\
\Delta t, N_{\exp }\end{array}$ & $\begin{array}{c}2015 \text { Feb } 26 \\
\Delta t, N_{\exp }\end{array}$ \\
\hline 3159 & \multicolumn{2}{|c|}{147.12} & 10.12 & $4.6,19$ & $4.5,28$ \\
\hline 3160 & \multicolumn{2}{|c|}{150.58} & 10.12 & $4.6,20$ & $4.5,28$ \\
\hline 3161 & \multicolumn{2}{|c|}{154.04} & 10.12 & $4.6,21$ & $4.5,28$ \\
\hline 3162 & \multicolumn{2}{|c|}{157.50} & 10.12 & $4.5,18$ & $4.5,28$ \\
\hline 3163 & \multicolumn{2}{|c|}{160.96} & 10.12 & $4.5,21$ & $4.6,28$ \\
\hline 3164 & \multicolumn{2}{|c|}{164.42} & 10.12 & $4.5,22$ & $4.6,28$ \\
\hline
\end{tabular}

Survey Observations in Late 2014 and Early 2015

Note. $\Delta \mathrm{t}$ is the time duration spanned by each observing set in hours and $N_{\text {exp }}$ is the total number of exposures for each night and field.

epoch. The constant values, $Z_{i}$, are introduced to correct the small aforementioned photometric zero-point variations. The least-squares minimization was applied to Equation (1) to obtain the other free parameters for each given $P$. The spin rate, $f$, was searched from 0.25 to $25 \mathrm{rev}_{\text {day }}{ }^{-1}$, with a step size of $0.025 \mathrm{rev} \mathrm{day}^{-1}$.

A quality code $(U)$ was then manually assigned to each folded light curve by visual inspection, where " 3 " means highly reliable; "2" means some ambiguity; and "1" means possible, but potentially wrong (Warner et al. 2009). Moreover, when no acceptable solution can be found for a light curve, it was assigned $U=0$. The uncertainty of the derived rotation period was estimated from periods with a $\chi^{2}$ smaller than $\chi_{\text {best }}^{2}+\Delta \chi^{2}$, where $\chi_{\text {best }}^{2}$ is the $\chi^{2}$ of the derived period and 
Table 2

Synodic Rotation Periods of PTF-U2s

\begin{tabular}{|c|c|c|c|c|c|c|c|c|c|c|c|c|c|c|c|c|c|}
\hline Obj ID & Designation & $a$ & $e$ & $i$ & $\Omega$ & $\omega$ & $\mathrm{D}$ & $\triangle$ & $r$ & $\alpha$ & $H_{R}$ & $n$ & $m$ & $\mathrm{PTF}_{R}$ & Period (hr) & $\Delta m$ & $\overline{\mathrm{U}}$ \\
\hline $00435^{\mathrm{a}}$ & (435) Ella & 2.45 & 0.16 & 1.82 & 23.2 & 333.6 & 23.3 & 2.69 & 1.74 & 7.00 & $10.18 \pm 0.13$ & 2 & 70 & $14.04 \pm 0.00$ & $4.64 \pm 0.02$ & 0.36 & 3 \\
\hline $00492^{\mathrm{a}}$ & (492) Gismonda & 3.11 & 0.18 & 1.62 & 46.2 & 296.5 & $59.9^{\mathrm{b}}$ & 3.66 & 2.68 & 0.90 & $9.68 \pm 0.07$ & 2 & 47 & $14.88 \pm 0.00$ & $6.44 \pm 0.04$ & 0.14 & 2 \\
\hline $00996^{\mathrm{a}}$ & (996) Hilaritas & 3.09 & 0.14 & 0.66 & 347.4 & 147.2 & $30.9^{\mathrm{b}}$ & 2.66 & 1.68 & 3.33 & $10.87 \pm 0.21$ & 7 & 68 & $14.25 \pm 0.00$ & $9.70 \pm 0.10$ & 0.54 & 2 \\
\hline $01223^{\mathrm{a}}$ & (1223) Neckar & 2.87 & 0.06 & 2.54 & 40.8 & 14.4 & $25.7^{\mathrm{b}}$ & 2.71 & 1.73 & 4.28 & $10.16 \pm 0.10$ & 3 & 42 & $13.89 \pm 0.00$ & $7.80 \pm 0.06$ & 0.21 & 2 \\
\hline $01635^{\mathrm{a}}$ & (1635) Bohrmann & 2.85 & 0.06 & 1.82 & 184.3 & 135.4 & $17.5^{\mathrm{b}}$ & 3.01 & 2.03 & 1.89 & $10.79 \pm 0.10$ & 2 & 69 & $14.96 \pm 0.00$ & $5.85 \pm 0.04$ & 0.34 & 3 \\
\hline
\end{tabular}

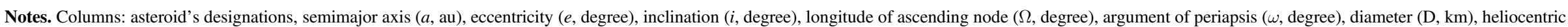

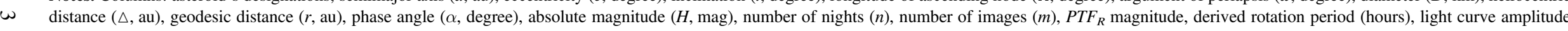
(mag) and rotation period quality code $(\mathrm{U})$. The full table is available in the electronic version.

${ }^{a}$ Asteroid available in the LCDB.

b WISE/NEOWISE diamer.

${ }^{\mathrm{c}}$ Light curves with large amplitudes and deep V-shape minima.

(This table is available in its entirety in machine-readable form.) 
Table 3

Asteroids with Partial Phase Coverage

\begin{tabular}{|c|c|c|c|c|c|c|c|c|c|c|c|c|c|c|c|c|c|}
\hline Obj ID & Designation & $a$ & $e$ & $i$ & $\Omega$ & $\omega$ & $\mathrm{D}$ & $\triangle$ & $r$ & $\alpha$ & $H_{R}$ & $\mathrm{n}$ & $\mathrm{m}$ & $\mathrm{PTF}_{R}$ & Period (hr) & $\Delta m$ & $\mathrm{U}$ \\
\hline $00870^{*}$ & (870) Manto & 2.32 & 0.26 & 6.19 & 120.8 & 196.9 & 13.0 & 2.91 & 1.93 & 3.29 & $11.45 \pm 0.12$ & 4 & 77 & $15.34 \pm 0.00$ & $30.00 \pm 4.05$ & 0.08 & 2 \\
\hline 01091 & (1091) Spiraea & 3.42 & 0.06 & 1.16 & 80.7 & 10.0 & $40.3^{w}$ & 3.28 & 2.32 & 4.74 & $10.70 \pm 0.08$ & 2 & 35 & $15.48 \pm 0.00$ & $7.01 \pm 0.43$ & 0.03 & 2 \\
\hline $01142^{*}$ & (1142) Aetolia & 3.18 & 0.08 & 2.11 & 139.3 & 96.2 & $24.4^{w}$ & 3.11 & 2.12 & 0.40 & $9.95 \pm 0.07$ & 2 & 48 & $14.05 \pm 0.00$ & $7.68 \pm 0.12$ & 0.15 & 2 \\
\hline $01782^{*}$ & (1782) Schneller & 3.11 & 0.16 & 1.54 & 157.4 & 107.2 & $21.9^{w}$ & 3.39 & 2.43 & 4.77 & $11.65 \pm 0.20$ & 3 & 43 & $16.59 \pm 0.01$ & $5.93 \pm 0.07$ & 0.71 & 2 \\
\hline 02142 & (2142) Landau & 3.16 & 0.12 & 0.66 & 155.5 & 34.2 & $20.1^{w}$ & 3.51 & 2.52 & 2.86 & $11.84 \pm 0.08$ & 3 & 55 & $16.86 \pm 0.01$ & $9.70 \pm 0.20$ & 0.28 & 2 \\
\hline
\end{tabular}

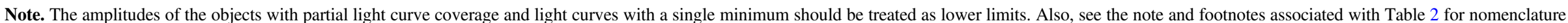
and explanations.

(This table is available in its entirety in machine-readable form.) 


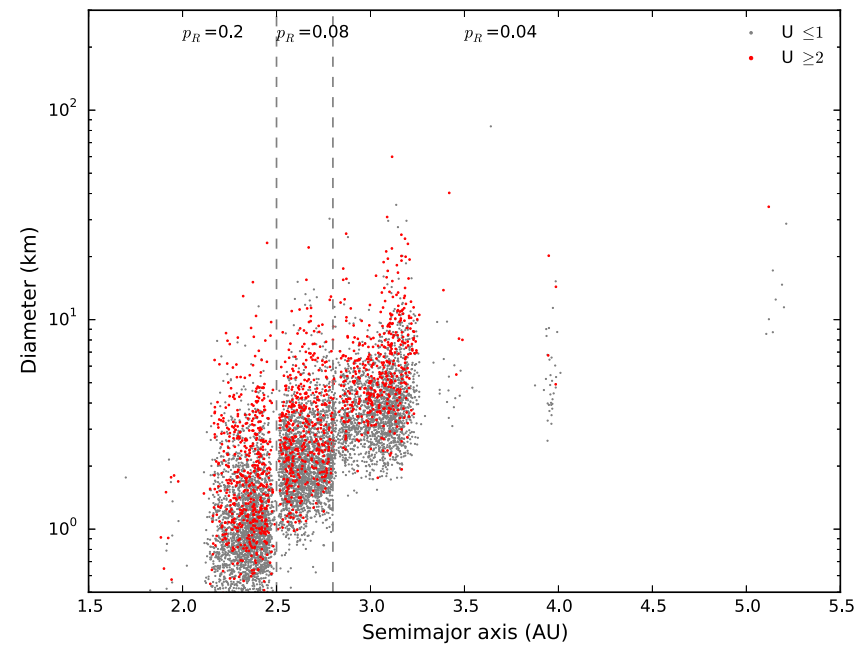

Figure 1. Diameters vs. semimajor axes for the PTF-U2s (red) and the PTFdetected asteroids (gray). The dashed lines show the divisions of empirical geometric albedo $\left(p_{R}\right)$ for asteroids located at different regions of the semimajor axis.

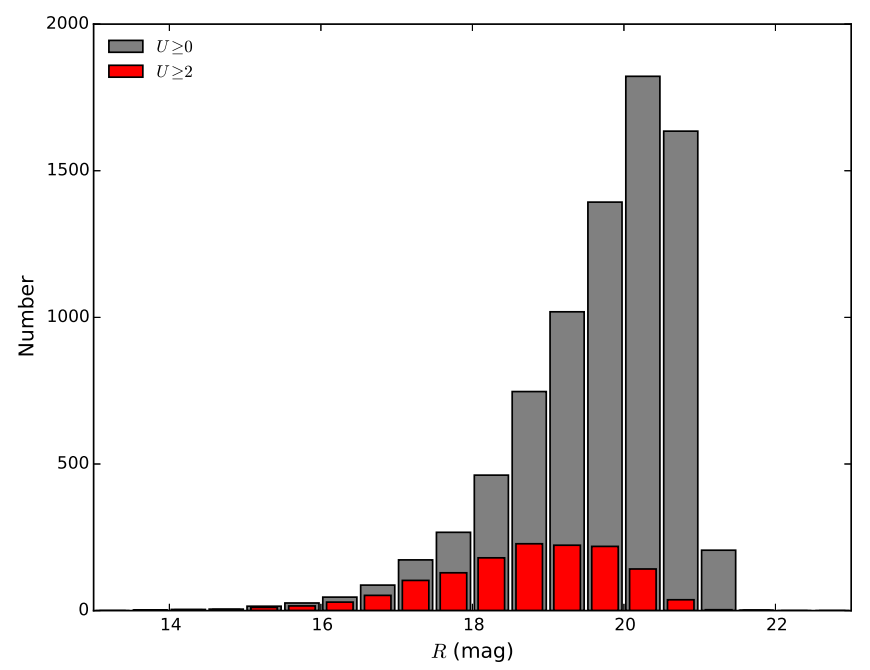

Figure 2. Magnitude distributions of the PTF-detected asteroids (gray) and the PTF-U2s (red) of this work.

$\Delta \chi^{2}$ is calculated from the inverse $\chi^{2}$ distribution, assuming $1+2 N_{k}+N_{i}$ degrees of freedom. We adopted the peak-topeak amplitude after rejecting the upper and lower $5 \%$ of data points to avoid outliers, which are probably contaminated by nearby bright stars or unfiltered artifacts during the light curve extraction.

Moreover, we adopted a WISE/NEOWISE diameter estimation, if available, for PTF-detected asteroids (Grav et al. 2011; Mainzer et al. 2011; Masiero et al. 2011). Otherwise, the diameter was estimated using

$$
D=\frac{1130}{\sqrt{p_{R}}} 10^{-H_{R} / 5},
$$

where $H_{R}$ is the $R$-band absolute magnitude, $D$ is the diameter in kilometers, $p_{R}$ is the $R$-band geometric albedo, and 1130 is the conversion constant adopted from Jewitt et al. (2013). Three empirical albedo values, $p_{R}=0.20,0.08$, and 0.04 , were assumed for asteroids in the inner $(2.1<a<2.5 \mathrm{au})$, mid

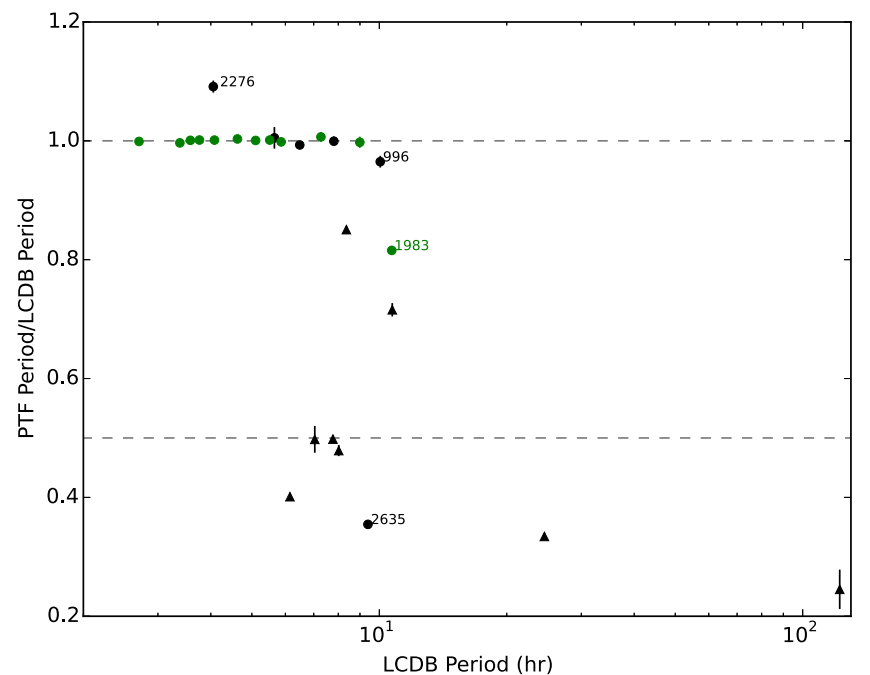

Figure 3. Comparison of 26 rotation periods of the PTF-U2s and the LCDB asteroids of $U=3$. Filled circles and filled triangles correspond to the PTF$\mathrm{U} 2 \mathrm{~s}$ and the PTF-Ps, respectively. Green and black indicate that the $U$ of the PTF-U2 is equal or worse than the matching LCDB object, respectively.

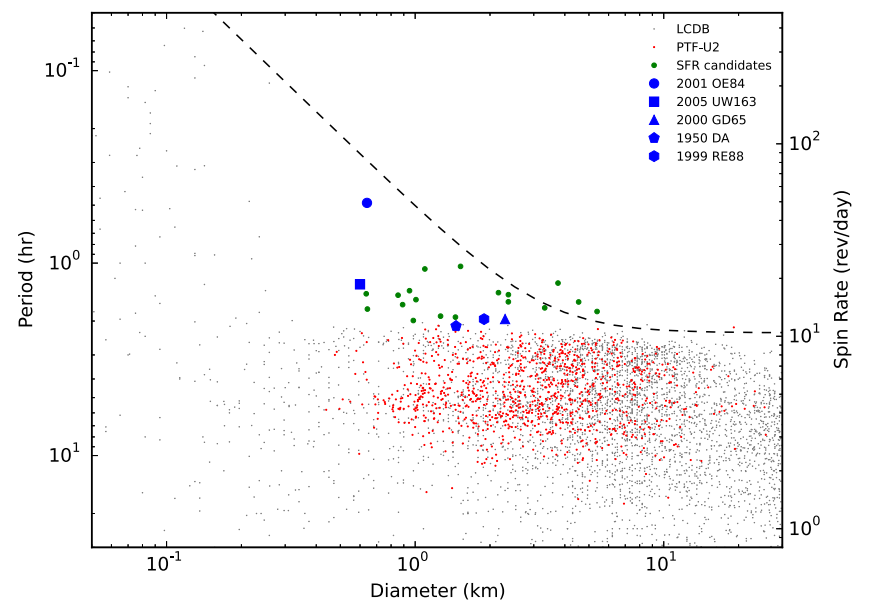

Figure 4. Asteroid rotation period vs. diameter. The red and gray filled circles are the PTF-U2s and LCDB objects of $U \geqslant 2$, respectively. The reported large SFRs are shown with larger blue filled symbols and the newly discovered large SFR, (40511) 1999 RE88, is represented by the blue filled hexagon. The green filled circles are the SFR candidates from this work. The dashed line is the predicted spin barrier adopted from Holsapple (2007). Note that the uncertainties in diameter estimation using Equation (2) for 18 SFR candidates are $\sim 10 \%$, according to the uncertainties in their absolute magnitude $H$.

$(2.5<a<2.8 \mathrm{au})$, and outer $(a>2.8 \mathrm{au})$ main belts, respectively (Tedesco et al. 2005).

\section{RESULTS AND DISCUSSION}

\subsection{Derived Rotation Periods}

We obtained 1029 reliable (i.e., $U \geqslant 2$ ) rotation periods (hereafter, PTF-U2s). Their rotation periods, orbital elements, and observational conditions are summarized in Table 2, and their folded light curves are given in Figure 10. Moreover, we also obtained 352 asteroids whose folded light curves show a clear trend, but do not fully cover one revolution (hereafter, PTF-Ps). Most of the PTF-Ps seem to have relatively long rotation periods (i.e., $f<2 \mathrm{rev}^{-1 a y}{ }^{-1}$ ) that cannot be recovered by our short observation time span. Therefore, their derived rotation periods can be treated as lower limits. These asteroids 


\begin{tabular}{|c|c|c|c|c|c|c|c|c|c|c|c|c|c|c|c|c|c|c|}
\hline Obj ID & Designation & $a$ & $e$ & $i$ & $\Omega$ & $\omega$ & $\mathrm{D}$ & $\triangle$ & $r$ & $\alpha$ & $H_{R}$ & $\mathrm{n}$ & $\mathrm{m}$ & $\mathrm{PTF}_{R}$ & Period (hr) & $\Delta m$ & $\mathrm{U}$ & $k$ \\
\hline 40511 & (40511) 1999 RE88 & 2.38 & 0.17 & 2.04 & 341.6 & 279.8 & $1.9^{w}$ & 2.61 & 1.62 & 1.93 & $16.36 \pm 0.30$ & 3 & 54 & $19.70 \pm 0.08$ & $1.96 \pm 0.01$ & 1.04 & 2 & 670 \\
\hline A0351 & (100351) 1995 SU88 & 2.42 & 0.13 & 0.64 & 356.5 & 199.4 & 1.0 & 2.71 & 1.72 & 1.37 & $17.05 \pm 0.20$ & 3 & 39 & $20.56 \pm 0.14$ & $1.99 \pm 0.01$ & 1.00 & 2 & 170 \\
\hline E4977 & (144977) 2005 EC127 & 2.21 & 0.17 & 4.75 & 336.9 & 312.8 & 0.9 & 2.45 & 1.46 & 1.58 & $17.27 \pm 0.19$ & 2 & 43 & $20.13 \pm 0.12$ & $1.64 \pm 0.01$ & 0.72 & 2 & 150 \\
\hline F2066 & (152066) 2004 PT108 & 2.56 & 0.20 & 2.28 & 335.7 & 287.2 & 2.2 & 2.82 & 1.87 & 7.01 & $16.33 \pm 0.25$ & 2 & 37 & $20.42 \pm 0.16$ & $1.42 \pm 0.01$ & 0.93 & 2 & 1740 \\
\hline G8089 & (168089) 2006 DM84 & 2.23 & 0.07 & 1.38 & 230.2 & 27.7 & 0.9 & 2.34 & 1.35 & 1.07 & $17.13 \pm 0.19$ & 1 & 24 & $19.79 \pm 0.09$ & $1.39 \pm 0.06$ & 0.68 & 2 & 220 \\
\hline J1530 & (191530) 2003 UX197 & 3.09 & 0.11 & 5.36 & 49.4 & 193.2 & 5.3 & 3.39 & 2.41 & 3.72 & $15.12 \pm 0.14$ & 3 & 36 & $20.01 \pm 0.13$ & $1.78 \pm 0.02$ & 0.50 & 2 & 2950 \\
\hline K02QC9J & 2002 QJ129 & 3.03 & 0.14 & 10.04 & 123.8 & 274.2 & 3.5 & 2.92 & 1.94 & 1.05 & $16.05 \pm 0.16$ & 3 & 64 & $19.95 \pm 0.11$ & $1.71 \pm 0.02$ & 0.51 & 2 & 1480 \\
\hline K05S16S & $\cdots$ & 2.70 & 0.09 & 1.22 & 222.4 & 181.1 & 1.3 & 2.46 & 1.48 & 3.23 & $17.50 \pm 0.18$ & 3 & 45 & $20.65 \pm 0.13$ & $1.89 \pm 0.01$ & 0.71 & 2 & 200 \\
\hline K08D80Z & $\cdots$ & 2.37 & 0.13 & 1.02 & 185.3 & 12.9 & 0.6 & 2.26 & 1.28 & 1.29 & $18.00 \pm 0.16$ & 2 & 45 & $20.46 \pm 0.12$ & $1.44 \pm 0.01$ & 0.63 & 2 & 80 \\
\hline K08UU6L & $\cdots$ & 3.07 & 0.16 & 4.01 & 278.7 & 141.4 & 2.4 & 2.79 & 1.81 & 1.67 & $16.89 \pm 0.15$ & 2 & 52 & $20.60 \pm 0.13$ & $1.59 \pm 0.01$ & 0.59 & 2 & 960 \\
\hline K15C40P & $\cdots$ & 3.07 & 0.03 & 9.52 & 149.9 & 82.7 & 3.8 & 3.04 & 2.05 & 0.82 & $15.89 \pm 0.18$ & 2 & 43 & $19.97 \pm 0.12$ & $1.27 \pm 0.01$ & 0.65 & 2 & 4650 \\
\hline L7411 & (217411) 2005 LD50 & 2.45 & 0.25 & 10.91 & 297.5 & 16.5 & 1.5 & 3.05 & 2.07 & 2.28 & $16.20 \pm 0.19$ & 2 & 60 & $20.41 \pm 0.13$ & $1.91 \pm 0.02$ & 0.73 & 2 & 270 \\
\hline L8602 & (218602) 2005 NE69 & 2.41 & 0.12 & 1.13 & 253.2 & 14.7 & 1.0 & 2.65 & 1.66 & 1.47 & $16.97 \pm 0.22$ & 3 & 55 & $20.38 \pm 0.14$ & $1.55 \pm 0.01$ & 0.86 & 2 & 260 \\
\hline P3384 & (253384) $2003 \mathrm{KQ} 3$ & 2.16 & 0.19 & 4.97 & 297.6 & 343.9 & 0.8 & 2.53 & 1.55 & 2.68 & $17.44 \pm 0.19$ & 3 & 51 & $20.68 \pm 0.14$ & $1.47 \pm 0.01$ & 0.71 & 2 & 160 \\
\hline P5828 & (255828) 2006 SC86 & 2.40 & 0.14 & 1.16 & 193.8 & 269.7 & 0.6 & 2.07 & 1.09 & 1.90 & $18.02 \pm 0.16$ & 3 & 64 & $19.88 \pm 0.09$ & $1.73 \pm 0.02$ & 0.47 & 2 & 40 \\
\hline Q8611 & (268611) 2006 CY30 & 2.73 & 0.03 & 6.34 & 169.6 & 181.7 & 2.4 & 2.81 & 1.84 & 4.56 & $16.14 \pm 0.15$ & 2 & 37 & $20.10 \pm 0.11$ & $1.46 \pm 0.01$ & 0.61 & 2 & 1240 \\
\hline W6242 & (326242) 2012 DS21 & 2.55 & 0.08 & 0.86 & 143.1 & 22.6 & 1.6 & 2.68 & 1.69 & 2.01 & $17.02 \pm 0.21$ & 3 & 52 & $20.45 \pm 0.13$ & $1.04 \pm 0.00$ & 0.75 & 2 & 1480 \\
\hline X2984 & (332984) 2011 FG67 & 3.19 & 0.04 & 13.17 & 32.0 & 75.8 & 4.5 & 3.14 & 2.17 & 4.29 & $15.47 \pm 0.16$ & 3 & 40 & $19.97 \pm 0.13$ & $1.59 \pm 0.01$ & 0.64 & 2 & 3630 \\
\hline b2963 & (372963) 2011 BY111 & 2.66 & 0.11 & 0.63 & 167.7 & 249.9 & 1.1 & 2.48 & 1.50 & 0.72 & $17.81 \pm 0.18$ & 2 & 42 & $20.64 \pm 0.14$ & $1.07 \pm 0.00$ & 0.66 & 2 & 590 \\
\hline
\end{tabular}

Note. The cohesion $k$ is calculated assuming a bulk density $\rho=2 \mathrm{~g} \mathrm{~cm}^{-3}$, except for (40511) 1999 RE88. Also, see the note and footnotes associated with Table 2 for nomenclature and explanations. 

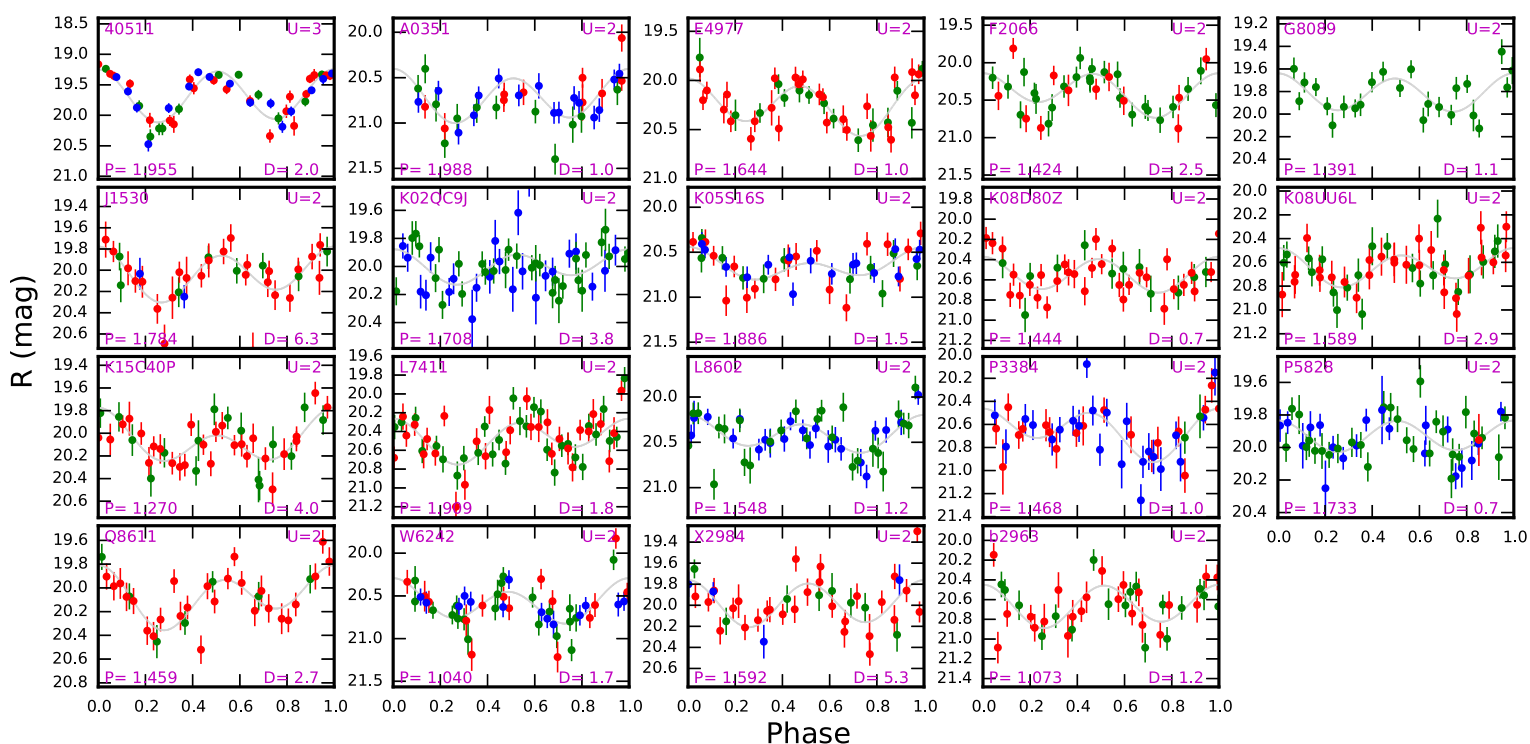

Figure 5. The 19 folded light curves for (40511) 1999 RE88, and other 18 candidates. The colors represent observations taken on different nights. The asteroid designation is given in each plot, along with its derived rotation period $P$ in hours and quality code $U$.

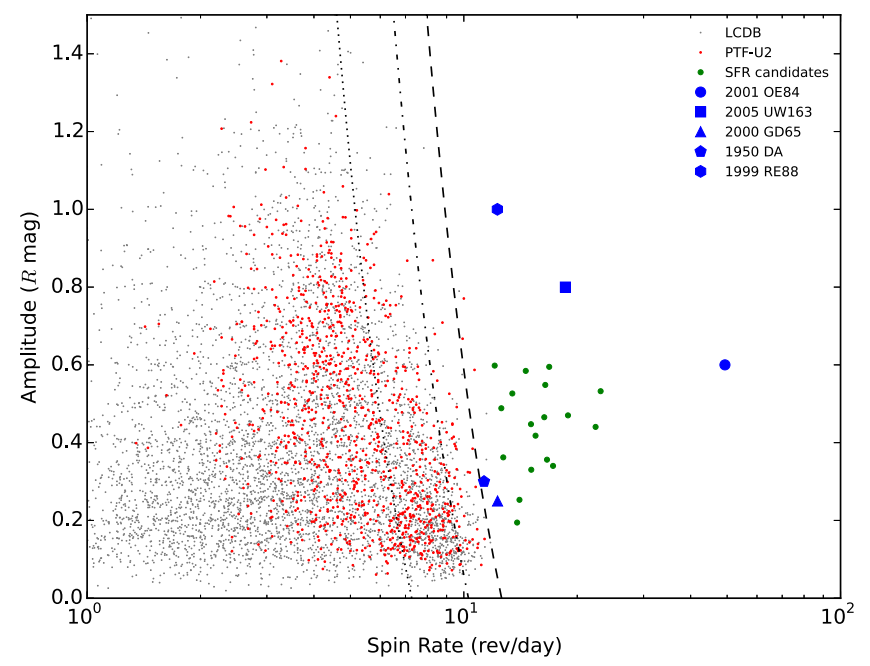

Figure 6. Light curve amplitude vs. spin rate. The symbols are the same with Figure 4. The dashed, dotted-dashed, and dotted lines represent the spin-rate limits for rubble-pile asteroids with bulk densities of $\rho=3,2$, and $1 \mathrm{~g} \mathrm{~cm}^{-3}$, respectively, according to $P \sim 3.3 \sqrt{(1+\Delta m) / \rho}$ (Pravec \& Harris 2000). Note that the asteroids of $D<0.2 \mathrm{~km}$ are not included in this plot.

are summarized in Table 3 and their folded light curves are given in Figure 11. Because of the survey area and the limiting magnitude, the majority of our samples are main-belt asteroids, as shown by the distribution of diameters versus semimajor axes in Figure 1. As expected, the chance of recovering the rotation period is better for brighter objects, as seen in Figure 2, which shows the overall magnitude distribution of the PTF-U2s and PTF-detected asteroids.

To examine our derived rotation periods, we compare our results with the $U=3$ asteroids in the LCDB. There are 26 overlapping objects, and the comparison is shown in Figure 3. In general, (a) 14 out of 17 derived rotation periods of the PTF-U2s are in good agreement with the LCDB values (i.e., the difference is $<3 \%$ ); (b) since the PTF-Ps have relatively large uncertainty in their derived rotation periods due to the incomplete light curve coverage on their full revolutions, all the PTF-Ps in Figure 3 show a certain degree of difference to the LCDB values; and (c) only two PTF-U2s, asteroid 996 and 2267, show minor differences (i.e., $\lesssim 10 \%$ ) and one PTF-U2, asteroid 2635, has a large difference with respect to the LCDB value. We discuss these three objects below. The $U$ codes of these three objects in our results were assigned as 2 , which means that these three objects have relatively large uncertainties in our results. In fact, our derived rotation periods for asteroid 996 (i.e., $9.70 \mathrm{hr}$ for the PTF-U2s and $10.05 \mathrm{hr}$ in the LCDB) and asteroid 2276 (i.e., $4.42 \mathrm{hr}$ for the PTF-U2s and $4.05 \mathrm{hr}$ in the LCDB) are in very good agreement with the LCDB values. However, our two-day observation time span was just long enough to merely cover the whole revolution of asteroid 996 and consequently leads to a shorter period. When we re-examined the periodogram for asteroid 2276, we found that its PTF light curve could be folded equally well on the periods of 4 and $4.8 \mathrm{hr}$, besides the best-fit $4.42 \mathrm{hr}$. The preference of $4.42 \mathrm{hr}$ is due to the resulting less dense light curve. When the observations were taken, asteroid 2635 happened to pass by a bright neighboring star and moved to the chip boundary. Consequently, most data points for asteroid 2635 were contaminated and are relatively unreliable. Moreover, it has a small light curve amplitude of $0.1 \mathrm{mag}$ (Mazzone 2012); we were therefore unable to identify its true rotation period. Overall, the derived rotation periods of the PTF$\mathrm{U} 2 \mathrm{~s}$ are reliable enough to yield statistics on the asteroid spin rate.

\subsection{Spin-rate Limit and Large Super-fast Rotators}

To investigate the spin-rate limit at $2.2 \mathrm{hr}$, we plot the diameters versus rotation periods of the PTF-U2s, along with that of the asteroids of $U=3$ in the LCDB. Figure 4 shows that most PTF-U2s are still below the $2.2 \mathrm{hr}$ limit, which is in accordance with the rubble-pile structure. However, 19 PTF$\mathrm{U} 2 \mathrm{~s}$ with diameters ranging from several hundred meters to several kilometers were located above the limit. We nominate these objects as large SFR candidates and list them separately in Table 4. Their folded light curves are given in Figure 5 and 

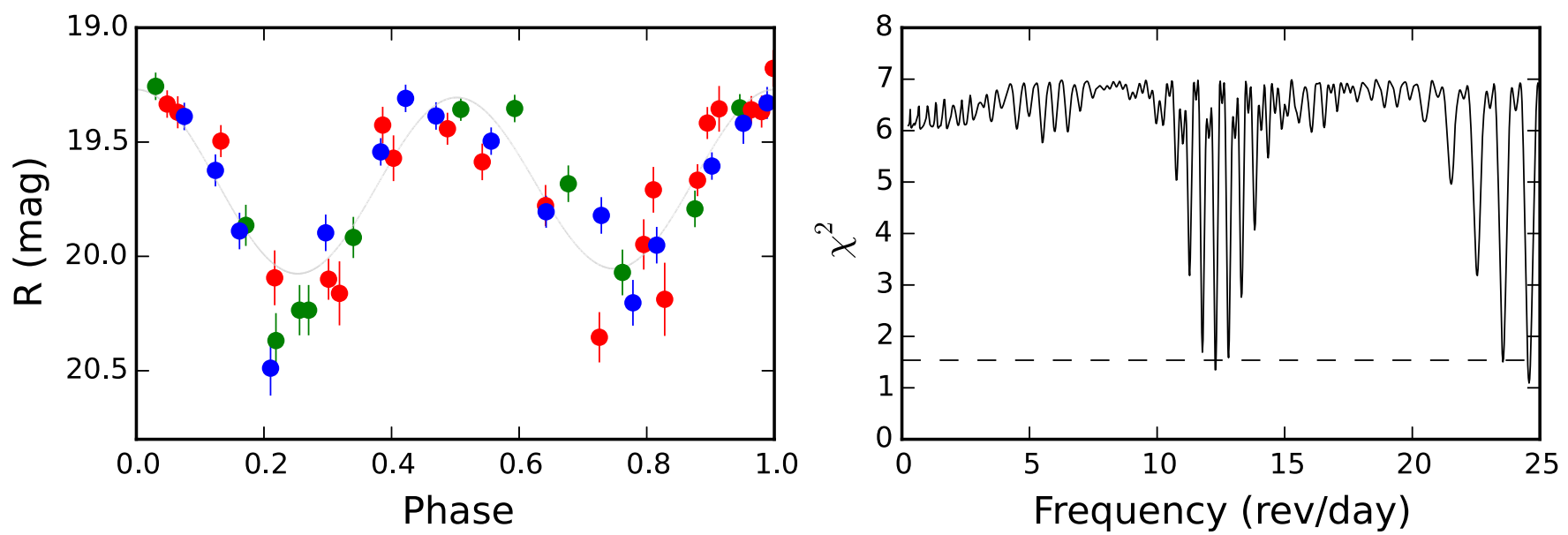

Figure 7. The folded light curve (left) and the periodogram (right) for the SFR (40511) 1999 RE88. Colors in the light curve represent observations taken on different nights. The dashed line in the periodograms indicates the uncertainties of the derived rotation periods. Note that an octahedron shape would have a maximum amplitude of $<0.4$ mag (Harris et al. 2014), and therefore the $\sim 1.0$ mag amplitude of (40511) 1999 RE88 can rule out the possibility of being at the double spin rate $f$ $=24.55 \mathrm{rev} \mathrm{day}^{-1}$.

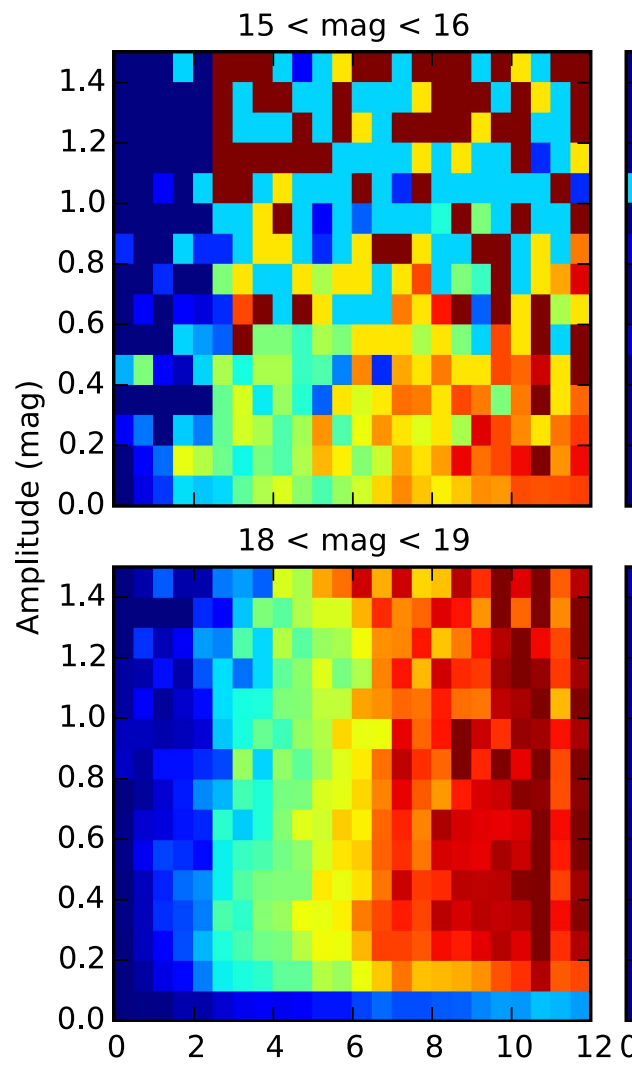

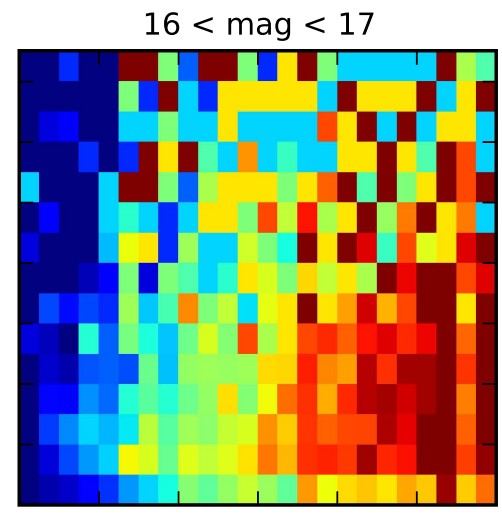
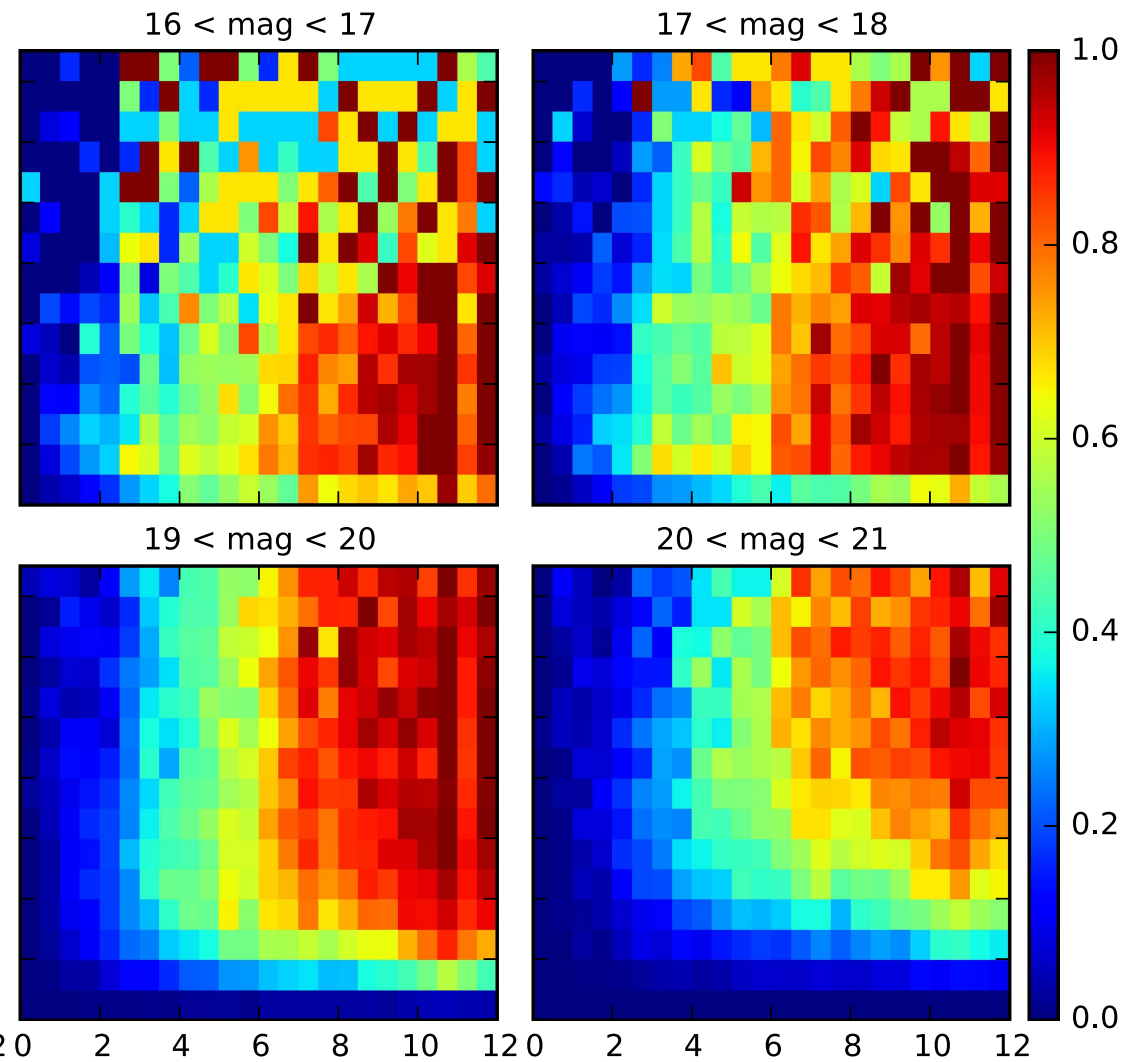

$20<\operatorname{mag}<21$

Frequency (rev/day)

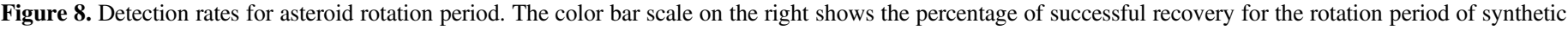
objects. The apparent magnitude interval is indicated on the top of each plot.

all show a clear trend. Asteroids with a diameter $D>150 \mathrm{~m}$ are believed to be rubble-pile, due to their complex collision history (Pravec et al. 2002). These large SFR candidates are of particular interest for understanding asteroid interior structure. When $P \sim 3.3 \sqrt{(1+\Delta m) / \rho}$ is applied to the PTF-U2s, the results suggest that these large SFR candidates have a bulk density of $\rho>3 \mathrm{~g} \mathrm{~cm}^{-3}$, as shown in the plot of spin rate versus amplitude in Figure 6. Such high bulk density is very unusual and it is therefore believed that internal cohesion might be present in asteroids (Holsapple 2007). However, the large SFRs, including these candidates, seem to comprise far less than the whole population of asteroids. This indicates that the large SFRs might be a special group aside from the "average" asteroids, which perhaps possess a different evolutionary history or mechanism for surviving under their super-fast rotations. 

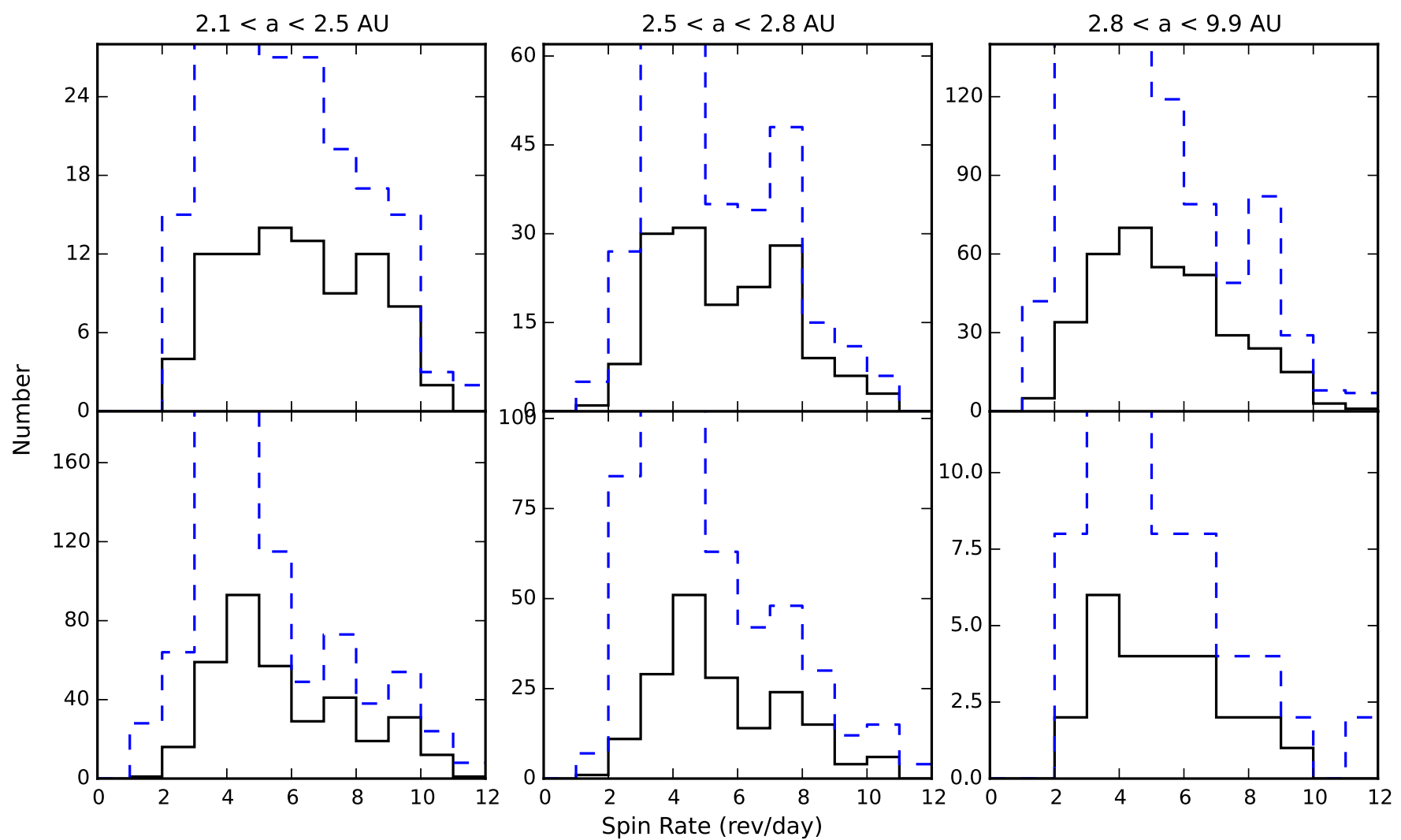

Figure 9. Spin-rate distributions for asteroids with diameters $3<D<15 \mathrm{~km}$ (top) and $D<3 \mathrm{~km}$ (bottom) at the inner (left), mid (middle) and outer (right) mainbelt. The black line and the blue dashed line are the original and de-biased results, respectively.

\subsubsection{The Large Super-fast Rotators: (40511) 1999 RE88}

Among the SFR candidates, the asteroid (40511) 1999 RE88 demonstrates a very clear folded light curve on the best-fit period of $1.96 \pm 0.01 \mathrm{hr}$ (see the left panel in Figure 7). When inspecting its periodogram, 1999 RE88 shows a simple profile with a very significant dip of $\chi^{2}$ at the best-fit frequency and a relatively high value of mean $\chi^{2}$ (see the right panel of Figure 7). This is very similar to the periodograms of other asteroids with $U=3$ in the PTF-U2s. The WISE/NEOWISE measurement gives it a diameter of $1.9 \pm 0.3 \mathrm{~km}$. Therefore, we identify (40511) 1999 RE88 as a newly discovered SFR. According to the SDSS color (i.e., $a^{*}=0.12 \pm 0.03$ and $i-z=0.64 \pm 0.11$ ) and the WISE/NEOWISE albedo (i.e., $p_{V}=0.18 \pm 0.04$ and $P_{\mathrm{IR}}=0.27 \pm 0.06$ ), this suggests that 1999 RE88 is an S-type inner main-belt asteroid. The folded light curve amplitude of $\sim 1$ mag rules out the possibility of an octahedron shape for 1999 RE88 (Harris et al. 2014). An asteroid with a diameter of 1999 RE88 is very unlikely to be monolithic, due to its complex collision history (i.e., $\sim 10^{4}$ impacts within $10^{9}$ years), as shown by Polishook et al. (2016). To calculate the internal cohesion that prevents 1999 RE88 from breaking apart in such fast rotation, we apply the DruckerPrager yield criterion (Holsapple 2007; Rozitis et al. 2014; Polishook et al. 2016):

$$
\begin{aligned}
& \frac{1}{6}\left[\left(\bar{\sigma}_{x}-\bar{\sigma}_{y}\right)^{2}+\left(\bar{\sigma}_{y}-\bar{\sigma}_{z}\right)^{2}+\left(\bar{\sigma}_{z}-\bar{\sigma}_{x}\right)^{2}\right] \\
& \quad \leqslant\left[k-s\left(\bar{\sigma}_{x}+\bar{\sigma}_{y}+\bar{\sigma}_{z}\right)\right]^{2}
\end{aligned}
$$

where $\left(\bar{\sigma}_{x}, \bar{\sigma}_{y}, \bar{\sigma}_{z}\right)$ are the three average orthogonal shear stresses, $k$ is the internal cohesion and $s$ is a slope constant determined by the angle of friction $\phi$, which was measured on lunar regolith as $40^{\circ}$ (Mitchell 1974)

$$
s=\frac{2 \sin \phi}{\sqrt{3}(3-\sin \phi)} .
$$

The $\left(\bar{\sigma}_{x}, \bar{\sigma}_{y}, \bar{\sigma}_{z}\right)$ can be calculated by

$$
\begin{gathered}
\bar{\sigma}_{x}=\frac{\left(\rho \omega^{2}-2 \pi \rho^{2} G A_{x}\right) a^{2}}{5}, \\
\bar{\sigma}_{y}=\frac{\left(\rho \omega^{2}-2 \pi \rho^{2} G A_{y}\right) b^{2}}{5}, \\
\bar{\sigma}_{z}=\frac{\left(-2 \pi \rho^{2} G A_{z}\right) c^{2}}{5},
\end{gathered}
$$

where $\rho$ is the bulk density, $\omega$ is the spin rate, $G$ is the gravitational constant, and $(a, b, c)$ are the axes of the asteroid ellipsoidal shape in which $a \geqslant b \geqslant c$. Moreover, the $\left(A_{x}, A_{y}\right.$, $A_{z}$ ) are dimensionless constants that depend on the axial ratios:

$$
\begin{aligned}
A_{x} & =\int_{0}^{\infty} \frac{d u}{(u+1)^{3 / 2}\left(u+\beta^{2}\right) 1 / 2\left(u+\alpha^{2}\right)^{1 / 2}}, \\
A_{x} & =\int_{0}^{\infty} \frac{d u}{(u+1)^{1 / 2}\left(u+\beta^{2}\right) 3 / 2\left(u+\alpha^{2}\right)^{1 / 2}}, \\
A_{x} & =\int_{0}^{\infty} \frac{d u}{(u+1)^{1 / 2}\left(u+\beta^{2}\right) 1 / 2\left(u+\alpha^{2}\right)^{3 / 2}},
\end{aligned}
$$




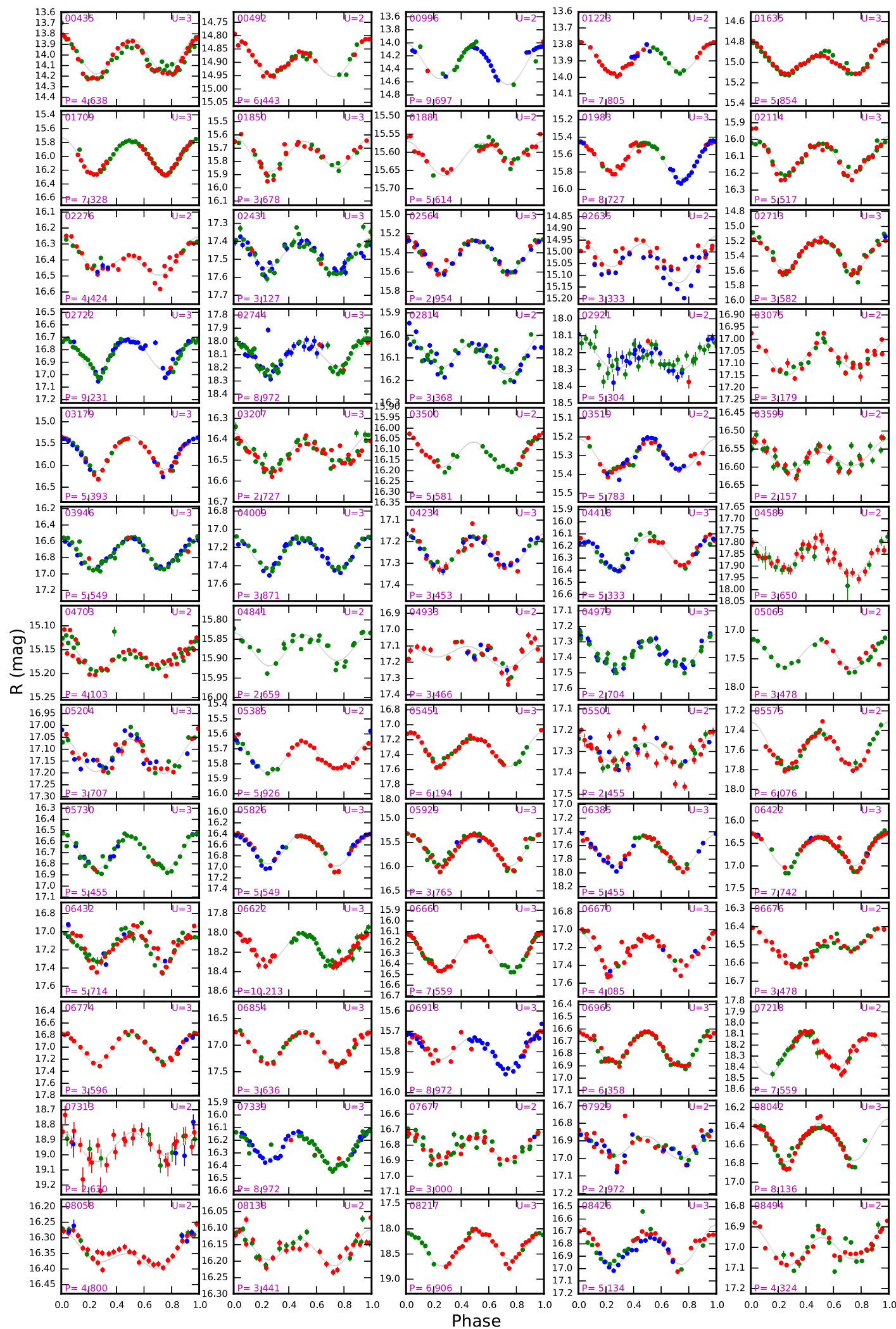

Figure 10. Set of 65 folded light curves for the PTF-U2s. The colors represent observations taken on different nights. The asteroid designation is given in each plot, along with its derived rotation period $P$ in hours and quality code $U$. The folded light curves for the remaining PTF-U2s are available in the extended figure in the electronic edition.

(An extended version of this figure is available.) 


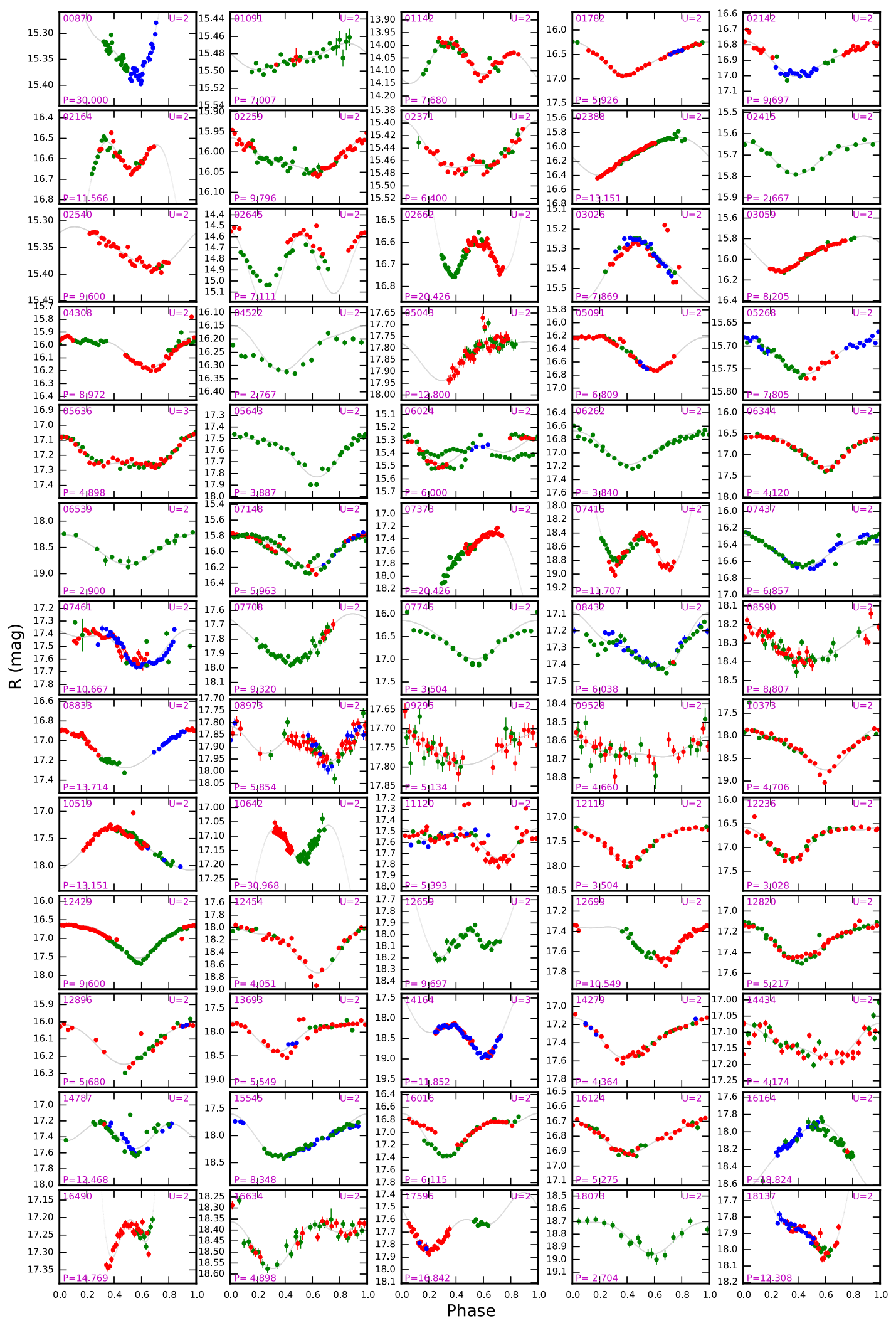

Figure 11. Set of 65 folded light curves for the PTF-Ps. The colors represent observations taken on different nights. The asteroid designation is given in each plot along with its derived rotation period $P$ in hours and quality code $U$. The folded light curves for the remaining PTF-Ps are available in the extended figure in the electronic edition.

(An extended version of this figure is available.) 
where $\alpha=c / a$ and $\beta=b / a$. We assume $a>b=c$, and use $\Delta M=1.0 \mathrm{mag}^{6}$ to calculate $a / b=2.51$ from $10^{0.4 \Delta M}$ for 1999 RE88. Using the average $\rho=2.72 \mathrm{~g} \mathrm{~cm}^{-3}$ for typical S-type asteroids (DeMeo \& Carry 2013), a cohesive strength of $780 \pm 500 \mathrm{~Pa}^{7}$ would be required to keep the fast-rotating 1999 RE88 intact. Combined with the cohesive strengths of other known SFRs (i.e., $2001 \mathrm{OE} 84, \sim 1500 \mathrm{~Pa}^{8}$; 2005 UW163, $\sim 200 \mathrm{~Pa}^{9}$; $2000 \mathrm{GD} 65,150$ to $450 \mathrm{~Pa}$ (Polishook et al. 2016; 1950 DA, $64 \mathrm{~Pa}$ (Rozitis et al. 2014)), all of them fall within the cohesion range of 100 to $1000 \mathrm{~Pa}$ of the lunar regolith (Mitchell 1974). This probably indicates the typical range of internal cohesion for asteroids. When assuming $\rho=2 \mathrm{~g} \mathrm{~cm}^{-3}$ for the 18 other SFR candidates, we found that 7 candidates with diameters of a few kilometers require $>1000 \mathrm{~Pa}$ cohesive strengths and the highest value can be up to $4000 \mathrm{~Pa}$ (see the last column in Table 4). Therefore, confirming the aforementioned SFR candidates, especially those requiring unusually large cohesive strengths, can provide important constraints on the asteroid interior structure.

\subsection{The Spin-rate Distribution}

In order to understand any possible observational bias in our survey, we followed the approach of Chang et al. (2015) to carry out a detection efficiency simulation (see Chang et al. (2015) and the references therein). Figure 8 shows the detection efficiency of spin rate versus light curve amplitude, in which we see that: (a) spin rates of $f \leqslant 3 \mathrm{rev}^{-1} \mathrm{y}^{-1}$ cannot be recovered; (b) the detection efficiency of $3<f<5$ rev day $^{-1}$ is $\lesssim 40 \%$, which is much lower than $\sim 90 \%$ in Chang et al. (2015); (c) the spin rates of the asteroids with small light curve amplitudes (i.e., $\Delta m<0.1 \mathrm{mag}$ ) are merely to be resolved; and (d) the detection efficiency decreases with increases of magnitude. The first two situations are due to our two-day observational time span, which hinders the recovery of relatively long rotation periods. The last two situations can be explained by the photometric uncertainty. When the asteroid's brightness variation is smeared in the photometric uncertainty, the rotation period is not likely to be recovered.

With this detection efficiency simulation, we generate the debiased spin-rate distributions and show them along with the original distributions in Figure 9, in which we separate the distributions according to asteroids' diameters (i.e., $3<D<15 \mathrm{~km}$ and $D<3 \mathrm{~km}$ ) and locations in the main belt (i.e., inner: $2.1<a<2.5 \mathrm{au}$, mid: $2.5<a<2.8 \mathrm{au}$, and outer: $a>2.8 \mathrm{au}$ ). Note that the PTF-Ps are not included in the statistics due to their relatively large uncertainties. As expected from the detection efficiency simulation, we see a significant underestimation in the number of $f \leqslant 3 \mathrm{rev}^{\mathrm{day}}{ }^{-1}$ and obvious differences between the original and de-biased results for $f \leqslant 5 \mathrm{rev}$ day $^{-1}$. Although the original distributions look different from Chang et al. (2015) (i.e., almost no objects

\footnotetext{
6 The amplitude of the light curve was not corrected for phase angle effects, due to its low-phase-angle (i.e., $<2$ degree) observations.

7 The uncertainty of the cohesion includes (a) the derived rotation period, in which we consider the other two solutions besides the best-fit solution as the upper/lower limits (i.e., $P=1.96 \pm 0.08 \mathrm{hr}$ ), (b) the light curve amplitude $\Delta m=1.0 \pm 0.1 \mathrm{mag}$, and (c) the WISE/NEOWISE diameter estimation $D=1.9 \pm 0.3 \mathrm{~km}$.

8 The cohesion is calculated here with the parameters in Pravec et al. (2002).

9 The cohesion is calculated here with the parameters in Chang et al. (2014b).
}

in $f \leqslant 2$ rev day $^{-1}$ and relatively less objects in $3 \leqslant f \leqslant 5 \mathrm{rev} \mathrm{day}^{-1}$ in this work), the de-biased results remain consistent in two ways: (a) for asteroids of $3<D<15 \mathrm{~km}$, the number in each spin-rate bin decreases along with increases of frequency for $f>5 \mathrm{rev} \mathrm{day}^{-1}$; and (b) for asteroids of $D<3 \mathrm{~km}$, a significant number drop at $f=5 \mathrm{rev}^{-1} \mathrm{day}^{-1}$ (i.e., the number of $f=6 \mathrm{rev}_{\text {day }}{ }^{-1}$ is only half of that in $f=5 \mathrm{rev} \mathrm{day}^{-1}$ ).

We aimed to discover large SFRs in this study and therefore, the observation time span of each campaign was chosen to be two days in order to obtain sky coverage that was as large as possible. Although this approach sacrificed the rotation period recovery rate, especially for relatively long periods, the quality of the spin-rate statistics still remains acceptable as a byproduct of our main goal.

\section{SUMMARY}

Five surveys for discovering large SFRs were carried out using the iPTF. Out of 1029 reliable rotation periods, we found 1 large SFR, (40511) 1999 RE88, and 1818 candidates. 1999 RE88 is an S-type inner main-belt asteroid with a diameter of $D=1.9 \pm 0.3 \mathrm{~km}$, which completes one rotation in $1.96 \pm 0.01 \mathrm{hr}$ and has a light curve amplitude of $\sim 1.0 \mathrm{mag}$. This requires an internal cohesion of $\sim 780 \mathrm{~Pa}$ for 1999 RE88 to remain intact under such fast rotation in the context of the rubble-pile model. Combining with other known large SFRs, their population seems to be relatively small compared to the entire asteroid population. This indicates that the large SFRs are probably a special group among asteroids.

Although the time span of just two days reduces the rotation period recovery, the spin rate distributions are in good agreement with the results of Chang et al. (2015), which show a number decrease along with increase of spin rate for asteroids with $3<D<15 \mathrm{~km}$ and a significant number drop at $f=6 \mathrm{rev}$ day $^{-1}$ for asteroids with $D<3 \mathrm{~km}$.

We would like to thank the anonymous referee for useful suggestions and comments. This work is supported in part by the National Science Council of Taiwan under the grants MOST 104-2112-M-008-014-MY3 and MOST 104-2119-M008-024, and also by the Macau Science and Technology Fund No. 017/2014/A1 of MSAR. This publication makes use of data products from WISE, which is a joint project of the University of California, Los Angeles, and the Jet Propulsion Laboratory/California Institute of Technology, funded by the National Aeronautics and Space Administration. This publication also makes use of data products from NEOWISE, which is a project of the Jet Propulsion Laboratory/California Institute of Technology, funded by the Planetary Science Division of the National Aeronautics and Space Administration. We gratefully acknowledge the extraordinary services that are specific to NEOWISE that are contributed by the International Astronomical Union's Minor Planet Center, operated by the Harvard-Smithsonian Center for Astrophysics, and the Central Bureau for Astronomical Telegrams, operated by Harvard University.

\section{REFERENCES}

Bowell, E., Hapke, B., Domingue, D., et al. 1989, Asteroids II (Tuscon, AZ: Univ. of Arizona Press), 21

Chang, C.-K., Ip, W.-H., Lin, H.-W., et al. 2014a, ApJ, 788, 17

Chang, C.-K., Ip, W.-H., Lin, H.-W., et al. 2015, ApJS, 219, 27 
Chang, C.-K., Lin, H.-W., \& Ip, W.-H. 2016, ApJ, 816, 71

Chang, C.-K., Waszczak, A., Lin, H.-W., et al. 2014b, ApJL, 791, LL35

DeMeo, F. E., \& Carry, B. 2013, Icar, 226, 723

Dermawan, B., Nakamura, T., \& Yoshida, F. 2011, PASJ, 63, 555

Grav, T., Mainzer, A. K., Bauer, J., et al. 2011, ApJ, 742, 40

Grillmair, C. J., Laher, R., Surace, J., et al. 2010, adass XIX, 434, 28

Harris, A. W. 1996, LPI, 27, 493

Harris, A. W., Pravec, P., Galád, A., et al. 2014, Icar, 235, 55

Harris, A. W., Young, J. W., Bowell, E., et al. 1989, Icar, 77, 171

Holsapple, K. A. 2007, Icar, 187, 500

Jewitt, D., Ishiguro, M., \& Agarwal, J. 2013, ApJL, 764, L5

Laher, R. R., Surace, J., Grillmair, C. J., et al. 2014, PASP, 126, 674

Law, N. M., Dekany, R. G., Rahmer, G., et al. 2010, Proc. SPIE, 7735, $77353 \mathrm{M}$

Law, N. M., Kulkarni, S. R., Dekany, R. G., et al. 2009, PASP, 121, 1395

Mainzer, A., Grav, T., Bauer, J., et al. 2011, ApJ, 743, 156

Masiero, J., Jedicke, R., Ďurech, J., et al. 2009, Icar, 204, 145

Masiero, J. R., Mainzer, A. K., Grav, T., et al. 2011, ApJ, 741, 68

Mazzone, F. 2012, http://www.astrosurf.com/salvador/Fotometria.html
Mitchell, J. K., Houston, W. N., Carrier, W. D., \& Costes, N. C. 1974, Apollo Soil Mechanics Experiment S-200 Final Rep. Space Sciences Laboratory Series 15, 7285 (Berkeley, CA: Univ. California Press)

Ofek, E. O., Laher, R., Law, N., et al. 2012a, PASP, 124, 62

Ofek, E. O., Laher, R., Surace, J., et al. 2012b, PASP, 124, 854

Polishook, D., \& Brosch, N. 2009, Icar, 199, 319

Polishook, D., Moskovitz, N., Binzel, R. P., et al. 2016, Icar, 267, 243

Polishook, D., Ofek, E. O., Waszczak, A., et al. 2012, MNRAS, 421, 2094

Pravec, P., \& Harris, A. W. 2000, Icar, 148, 12

Pravec, P., Harris, A. W., Vokrouhlický, D., et al. 2008, Icar, 197, 497

Pravec, P., Kušnirák, P., Šarounová, L., et al. 2002, in Asteroids, Comets, and Meteors: ACM Vol. 500, ed. B. Warmbein (Noordwijk: ESA), 743

Rau, A., Kulkarni, S. R., Law, N. M., et al. 2009, PASP, 121, 1334

Rozitis, B., Maclennan, E., \& Emery, J. P. 2014, Natur, 512, 174

Rubincam, D. P. 2000, Icar, 148, 2

Sánchez, D. P., \& Scheeres, D. J. 2012, Icar, 218, 876

Tedesco, E. F., Cellino, A., \& Zappalá, V. 2005, AJ, 129, 2869

Warner, B. D., Harris, A. W., \& Pravec, P. 2009, Icar, 202, 134

Waszczak, A., Chang, C.-K., Ofek, E. O., et al. 2015, AJ, 150, 75

York, D. G., Adelman, J., Anderson, J. E., Jr., et al. 2000, AJ, 120, 1579 